\title{
Professor Xiaoquan Du's Seven Methods of Treating Stomachache
}

\author{
Xuzhao Wang1*, Li Liu', Xiaoquan Du1, 2, Chunxia Ma1, Wei Cui ${ }^{1}$ \\ 1Shaanxi University of Chinese Medicine, Xianyang 712046, Shaanxi Province, China \\ 2The First Affiliated Hospital of Shaanxi University of Chinese Medicine, Xianyang 712046, Shaanxi Province, China \\ *Corresponding author: Xuzhao Wang, 1016911627@qq.com
}

\begin{abstract}
Stomachache is the main symptom of stomach duct pain near the heart. Professor Xiaoquan Du is experienced in treating spleen and stomach diseases with traditional Chinese medicine (TCM) syndrome differentiation. He has vast practical TCM experience through years of clinical diagnosis and treatment, and incorporated his own characteristic methods in treatments, thereby developing seven methods for the treatment of stomachache. Clinically, methods such as "invigorating the spleen and benefiting the stomach" and "warming the middle and dispersing cold" are mostly adopted to treat stomach duct pain.
\end{abstract}

Keywords: Stomachache; Treatment; Traditional Chinese medicine

Publication date: July 2021; Online publication: July 30, 2021

\section{Etiology and pathogenesis}

Stomach duct pain is called "stomach heart pain" in ancient time. Stomach pain is first noted in "The Internal Canon of Medicine," "Magic Pivot, Forms of Disease from Evil Qi in the Bowels and Viscera," and "Man with Stomach Disease, Feel Pain in Stomach Heart. "1]" Stomachache in the upper abdomen near the pit of the heart, or associated with the ribs, including burning, spasmodic pain, dull pain, swelling pain, tingling pain, and pain, can occur before or after eating ${ }^{[2]}$. From the angle of traditional Chinese medicine (TCM), it is believed that the cause of the disease is related to emotion, diet and season. Stomachache happens in the stomach, and is related to the liver and spleen. The pathological nature of stomachache is deficiency and excess. Deficiency syndrome is mainly the deficiency of qi, blood, yin and yang in the spleen and stomach, and excess syndrome is often accompanied by evil qi, such as cold, heat, dampness, blood stasis and food accumulation. From the perspective of modern Western medicine, it is believed that the occurrence of stomach duct pain is related to acute and chronic gastritis, gastroduodenal ulcer, oesophagitis, gastric cancer and other diseases ${ }^{[3]}$. Professor Xiaoquan Du thinks that the daily life of modern people is irregular, their diet is unclean, and their spleen and stomach are damaged. If they have insufficient intake of nutrients or are physically weak, their spleen and stomach will also become weak; insufficiency of the source of transformation will occur, and they will feel pain if they are not flourish. Overeating or irregular diet will increase the burden of spleen and stomach transportation, and cause damp heat, stagnation, blood stasis block, and impassable pain.

\section{Seven special treatment methods for stomachache}

\subsection{Method of invigorating spleen and stomach}

By tonifying the $q i$ of spleen and stomach, the digestive and absorption functions of spleen and stomach can be enhanced. This treatment method is suitable for lessen stomachache, fatigue and weakness, loss of 
appetite, stomach duct pain caused by eating too little, and pain with eating, thin tongue, thin white fur, weak pulse, etc. Six gentlemen soup for center fortification is usually used to cure the deficiency. Medications, such as Radix Codonopsis, Radix Astragali seu Hedysari, Rhizoma Atractylodis Macrocephalae, and Poria cocos fuling tuckahoe, are used. Professor Xiaoquan Du thinks that deficiency of $q i$ and blood in spleen and stomach is the root of pathogenesis, and other syndromes can be developed on this basis. In the process of treating stomachache, as long as dampness and heat, blood stasis and other evil qi are removed, the method of invigorating spleen and stomach and replenishing $q i$ and blood should be used as soon as possible. Second, it is important to strengthen the function of the spleen and stomach, generate more $q i$ and blood, and nourish other viscera. Third, the spleen and stomach are the hub of human body $q i$. Spleen directs $q i$ upwards and stomach directs $q i$ downwards, causing all the $q i$ of the body to be circumfluent, in order to fundamentally prevent the occurrence and progression of the disease.

\subsection{Method of warming the center and dissipating cold}

This method uses warm medicine to nourish the stomach, so that the cold in stomach can be removed. When yang is exuberant, gastric peristalsis and emptying should be used. It is suitable for stomach cold syndrome. Since stomach cold and dust pain may often occur, stomach would fall trap to cold, and have a preference for heat. Professor Xiaoquan Du's method embodies two aspects: it is necessary to distinguish between the syndrome of excessive cold syndrome and deficiency cold syndrome. When the symptoms are not obvious and difficult to distinguish, Professor Xiaoquan Du often considers excessive cold and deficiency cold according to the deficiency and excessive of tongue veins. The treatment of excessive cold is mainly to dispel cold evil. The commonly used medications are Lesser Galangal and Cyperus Pill, which can be added with other drugs such as Herba Ephedrae and Ramulus Cinnamomiand, in order to remove the cold evil. The deficiency cold was treated with major and minor Center-Fortifying Decoction to warm the center and remove cold.

\subsection{Method of Soothing Liver and Stomach}

By using the drugs of dispersing liver $q i$ and regulating $q i$, the function of $q i$ to regulate esophagus, stomach and intestine can be improved. It is suitable for the syndrome of disharmony between liver and stomach, manifested as occasional distention of stomach duct, stomach duct pain spreading to hypochondrium, and belching, which are related to emotion and wiry pulse. This can be treated with Bupleurum Liver-Coursing Powder and Jieyu Hehuan soup. Professor Xiaoquan Du believes that this method emphasizes "harmony," so that the wood and soil perform their own duties, do not offend each other, and use drugs to achieve a balanced state of liver and stomach.

\subsection{Method of nourishing stomach yin}

This method nourishes the stomach, so that qi, blood and yin moisten the stomach, and promote gastric juice secretion. It is suitable for the syndrome of deficiency of stomach yin, manifested as epigastric dull pain, burning pain, dry mouth, loss of appetite, and thin, red or light red tongue, etc. Adenophora/glehnia and Ophiopogon Decoction can be used. Professor Xiaoquan Du likes to use Rhizoma Phragmitis and Rhizoma Anemarrhenae to enter the lung meridian and kidney meridian. Rhizoma Phragmitis irrigates and diffuses the body fluid, while Rhizoma Anemarrhenae makes the yin fluid transpiration up and down to nourish the yin of the Middle Earth (中土, transliterated zhong tu).

\subsection{Method of removing blood stasis and clearing collaterals}

This method is to reduce the blood collaterals in the stomach and stasis, so that the meridians and collaterals of the stomach and the whole body are unobstructed. It is suitable for the syndrome of stasis of gastric 
collaterals, which is manifested as chronic stomachache, pain at fixed position, dull pain, tingling pain, black stools, and purple tongue. Commonly used drugs include Salvia Beverage drink and Sudden Smile Powder. In the treatment of this syndrome, Professor Xiaoquan Du likes to add some products to strengthen the spleen and replenish $q i$, such as Radix Codonopsis and Endothelium Corneum Gigeriae Galli, which can remove blood stasis without damaging people's healthy $q i$, and can strengthen the circulation of $q i$ and blood, so that blood stasis is removed more quickly.

\subsection{Method of inhalation and gastric}

This method eliminates food stagnation and helps with the digestion of the spleen and stomach. It is suitable for food stagnation and blocking syndrome, which is caused by improper diet, resulting in stomach pain and fullness, loss of appetite, belching and acid, white and greasy tongue coating. Yellow and greasy tongue coating mayindicate food accumulation heat. Commonly used drug is Harmony-Preserving Pill. Professor Xiaoquan Du believes that it is not appropriate to use tonic and greasy drugs for this disease as they will cause obstruction. Although patients have weakness and dry mouth, they should use tonic method according to the symptoms after removing the evil $q i$ of food accumulation.

\subsection{Method of clearing dampness for gastric digestion}

By removing the dampness in the stomach, the stomach $q i$ is flourishing, the appetite is increased, and the function of decomposability is restored. It is suitable for treating dampness and turbidity syndrome. Symptoms of this syndrome include epigastric bulge, dull pain, loss of appetite, the feeling of not wanting to drink water, mouth stickiness and tastelessness, drowsiness, and white and greasy tongue fur. This syndrome can be treated with Stomach-Calming Powder and Agastache, and Qi-Righting Powder. Professor Xiaoquan Du believes that medication must be taken until the fur on the tongue is completely removed, as changes in the tongue pattern can accurately reflect the condition.

\section{Summary}

Stomachache is very common in our life and we should be alert to this illness whenever it happens. At present, Western medicine recommends taking proton pump inhibitors and anti-Helicobacter pylori drugs, but due to inter-individual differences, patient satisfaction is not high, and the use of medication is not as flexible as that in $\mathrm{TCM}^{[4]}$. The treatment of stomach dust pain based on syndrome differentiation of TCM has a unique curative effect. Different programs and drugs can be formulated according to patients, which is more personalized to the needs of patients. Therefore, the use of TCM or the combination of traditional Chinese and western medicine is the future trend. Professor Xiaoquan Du believes that the treatment of stomachache should first, distinguish deficiency from excess, and second, identify cold from heat. The course of the stomachache is long, and it might involve both cold and heat. Pathological factors such as hot and humid, stagnancy, and blood stasis are often mixed with each other. This condition is commonly seen in the clinical treatment and medication is given when these factors are at play. Seven kinds of treatment are often used. For example, blood stasis stomachache can be caused by the deficiency of the spleen and stomach of the middle Jiao, and stagnation of the blood collagals and poor operation. Therefore, warming the middle and dispersing cold is the method to cure the root cause. With the combination of promoting blood circulation, removing blood stasis and treating symptoms, good results can be achieved. In addition, we should still pay attention to therapeutics of affective diet, and encourage the patient to practice regular and balanced diet work and rest, and to relax.

\section{Disclosure statement}

The authors declare that there is no conflict of interest. 


\section{References}

[1] Qiu Y, Feng X, 2018, Clinical Research Status of Acupuncture in the Treatment of Epigastric Pain. Asia-Pacific Traditional Medicine, 14(1): 120-122.

[2] Diagnostic Basis, Syndrome Classification and Curative Effect Evaluation of Epigastric Pain - The Standard of TCM Diagnosis and Curative Effect of TCM Internal Disease Syndrome (ZY/T001.1-94) of the People's Republic of China. Journal of Practical Traditional Chinese Internal Medicine, 2021, 35(2): 128.

[3] Li J, 2019, Clinical Observation on 70 Cases of Stomach Duct Pain with Helicobacter pylori Infection Treated with Stomach-Calming Powder [J]. Clinical research of traditional Chinese medicine, 11(25): 101-103.

[4] Wu J, Gan H, Wu H, et al., 2017, Research Progress of TCM Syndrome Differentiation and Classification in the Treatment of Epigastric Pain. Asia-Pacific Traditional Medicine, 13(8): 84-85. 